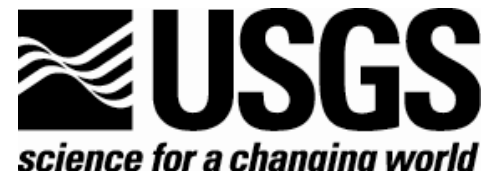

\title{
Ostracode Analysis for Cores BL96-1 and BL96-2 from Bear Lake, Utah and Idaho
}

By Jordon Bright, Richard Forester, and Darrell S. Kaufman

Open-File Report 2005-1227

U.S. Department of the Interior

U.S. Geological Survey 


\section{U.S. Department of the Interior \\ Gale A. Norton, Secretary}

\section{U.S. Geological Survey \\ Charles G. Groat, Director}

U.S. Geological Survey, Reston, Virginia 2005

For sale by U.S. Geological Survey, Information Services

Box 25286, Denver Federal Center

Denver, CO 80225

For more information about the USGS and its products:

Telephone: 1-888-ASK-USGS

World Wide Web: http://www.usgs.gov/

Any use of trade, product, or firm names in this publication is for descriptive purposes and does not imply endorsement by the U.S. Government.

Although this report is in the public domain, permission must be secured from the individual copyright owners to reproduce any copyrighted material contained within this report.

This report has not been reviewed for stratigraphic nomenclature.

Suggested citation:

Bright, J., Forester, R.F., and Kaufman, D.S., 2005, Ostracode analysis for cores BL96-1 and BL96-2 from Bear Lake, Utah and Idaho: U.S. Geological Survey Open-File Report 2005-1227, 13p.

Prepared by the U.S. Geological Survey in Denver, Colorado (http://climchange.cr.usgs.gov/) 


\section{Ostracode Analysis for Cores BL96-1 and BL96-2 from Bear Lake, Utah and Idaho}

By Jordon Bright, Richard Forester, and Darrell S. Kaufman

\section{Introduction}

This report summarizes the results of ostracode faunal analyses of two cores, 96-1 and 96-2, located in depth of 50 and $40 \mathrm{~m}$, respectively, from Bear Lake, Utah and Idaho. The lower third of core 96-2 is composed of reddish carbonate-poor fine-grained sediment. This siliciclastic material is overlain by gray carbonate-rich mud (Dean and others, 2005). The deposition rate at the site of core 96-1 was higher than at the site of core 96-2 (Colman and others, 2005), and all of the section sampled by core 96-1 corresponds roughly to the upper half of the carbonate-rich sediments in core 96-2. This report primarily describes the ostracode data and secondarily discusses the stratigraphic structure of the ostracode species profiles. These data contribute to understanding the environmental history of Bear Lake (for example, Schwalb and others, 1995; Smith, 1993; Forester, 1987).

\section{Methods}

Samples from core 96-1 were analyzed for ostracodes prior to amino acid analysis (Kaufman, 2003) at Northern Arizona University (NAU). Samples were weighed moist, then disaggregated for several days in deionized water before washing over a 100 mesh $(150 \mu \mathrm{m})$ sieve to retrieve the ostracode valves. The residues were air dried in the sieves, and whole adult ostracode valves were counted (whole adults that showed minor damage were included in the count). Partial valves were not counted. A total of 1,618 adult valves were counted from 22 core intervals.

Core 96-2 was sampled systematically at intervals of 4 centimeters. The samples were prepared at the U.S. Geological Survey in Denver, Colorado, and reference slides were sent to NAU for ostracode counts. Slide contents were dry sieved at 50 mesh $(300 \mu \mathrm{m})$ and 100 mesh $(150 \mu \mathrm{m})$ to separate the adult Candona valves from the Limnocythere adults. Only whole, adult valves were counted. A total of 5,753 adult valves were counted from 99 core intervals. Representative male and female valves of each species were photographed using a scanning election microscope. Digital versions of these images are in the Appendix. 


\section{Results}

Ostracodes were abundant and well preserved through core 96-1 and the upper carbonate-rich two thirds of core 96-2 (table 1). With the exception of one taxon found in core 96-1, all of the taxa are undescribed species and, therefore, appear to be endemic to Bear Lake. In contrast, ostracodes collected from the littoral zone of Bear Lake are common species currently found in streams and marshes throughout the Bear Lake valley (Bright, unpublished data). The rich fauna (10 endemic species) in Bear Lake is inconsistent with other large North American lakes. For example, only three ostracode species typify the deep-lake sediments of Lake Huron (Dettman and others, 1995) and Lake Michigan (Forester and others, 1994). This may be due to the young $(<12,000 \mathrm{yr})$ age of these lakes. By comparison, nearly 200 endemic species of ostracodes are known from Lake Tanganyika, Africa, which ranks second in species diversity behind Lake Baikal, Russia (Wells and others, 1999). The high diversity in these lakes probably is related to their longevity (9-12 Ma) and complex habitat structures within the lakes (Wells and others, 1999; Forester, 1991). If so, then the endemic fauna of Bear Lake hints at a lake considerably older or more complex than typical deep North American lakes.

Table 1. Ostracodes found in Bear Lake cores 96-1 and 96-2.

$\mathrm{X}$ indicates species is present.

-- indicate species absent

\begin{tabular}{|c|c|c|}
\hline & 96-1 & 96-2 \\
\hline \multirow[t]{2}{*}{ Candona sp. 1} & & $\mathrm{X}$ \\
\hline & $\mathbf{X}$ & \\
\hline Candona sp. 2 & $\mathrm{X}$ & $\mathrm{X}$ \\
\hline Candona sp. 3 & $\mathrm{X}$ & $\mathrm{X}$ \\
\hline Candona sp. 4 & $X$ & $\mathrm{X}$ \\
\hline Candona sp. 5 & $\mathrm{X}$ & $\mathrm{X}$ \\
\hline Candona sp. 6 & $\mathrm{X}$ & $\mathrm{X}$ \\
\hline Candona sp. 7 & $\mathrm{X}$ & $\mathrm{X}$ \\
\hline Limnocythere sp. 1 & $\mathrm{X}$ & $\mathrm{X}$ \\
\hline Limnocythere sp. 2 & -- & $\mathrm{X}$ \\
\hline unidentified & $\mathrm{X}$ & $\mathrm{X}$ \\
\hline Ilyocypris bradyi & $X$ & -- \\
\hline
\end{tabular}

\section{Core 96-1}

Core 96-1 contains 10 ostracode species from four genera (table 2). The genus Candona is represented by seven species, three of which are common. The genera Limnocythere and Ilyocypris are each represented by one species, and there is one unidentified species. Ostracode valve data from core 96-1 primarily were intended for amino acid analyses. Only the common taxa were enumerated, and their distributions are shown on figure 1.

\section{Core 96-2}

Core 96-2 also contains 10 species from three genera (table 3). Candona is represented by seven species, four of which are common. The seven Candona species are the same species found in core 96-1. Limnocythere is represented by the same species as core 96-1 and by an isolated occurrence of a second species. An unidentified species is present as well. The occurrence of each species is summarized on figure 2 . 
Table 2. Ostracode concentrations in core 96-1.

Note: The core was cut into 5 sections, A through E. Sample numbers indicate the depth in each section, in centimeters, relative to the top of the core liner for that section. The Depth in the core is the depth relative to the uppermost sediments in section $A$

Sample No. Depth Candona sp1 Candona sp.2 other Candona Limno.. sp 1 unident.

\section{Total}

\begin{tabular}{cccccccc} 
& $(\mathrm{cm})$ & $($ valves $/ \mathrm{g})$ & (valves $/ \mathrm{g})$ & (valves $/ \mathrm{g})$ & (valves $/ \mathrm{g})$ & (valves /g) & (valves /g) \\
\hline A-7 & 3 & 1.9 & 1.1 & 1.1 & 0.0 & 0.0 & $\mathbf{4 . 1}$ \\
A-52 & 48 & 4.3 & 2.2 & 9.0 & 11.1 & 2.9 & $\mathbf{2 9 . 5}$ \\
A-70 & 66 & 2.8 & 1.0 & 0.0 & 0.0 & 0.0 & $\mathbf{3 . 8}$ \\
A-81 & 77 & 4.1 & 7.4 & 2.6 & 0.0 & 1.5 & $\mathbf{1 5 . 6}$ \\
A-91 & 87 & 2.6 & 2.6 & 0.5 & 0.0 & 0.0 & $\mathbf{5 . 7}$ \\
B-29 & 126 & 7.2 & 33.2 & 7.2 & 0.0 & 0.4 & $\mathbf{4 8 . 0}$ \\
B-49 & 146 & 3.9 & 9.6 & 7.3 & 2.2 & 4.7 & $\mathbf{2 7 . 7}$ \\
B-69 & 166 & 1.5 & 2.5 & 1.0 & 1.6 & 0.1 & $\mathbf{6 . 7}$ \\
B-89 & 186 & 1.4 & 0.4 & 2.2 & 1.0 & 0.4 & $\mathbf{5 . 4}$ \\
B-99 & 196 & 0.3 & 0.0 & 1.0 & 0.0 & 0.0 & $\mathbf{1 . 3}$ \\
C-29 & 226 & 0.5 & 0.1 & 0.1 & 0.4 & 0.1 & $\mathbf{1 . 2}$ \\
C-49 & 246 & 2.3 & 4.0 & 3.1 & 0.7 & 1.4 & $\mathbf{1 1 . 5}$ \\
C-69 & 266 & 2.9 & 5.7 & 1.4 & 1.3 & 0.3 & $\mathbf{1 1 . 6}$ \\
C-99 & 296 & 3.1 & 0.4 & 3.2 & 0.8 & 1.1 & $\mathbf{8 . 6}$ \\
D-29 & 327 & 0.2 & 0.0 & 0.1 & 0.0 & 0.0 & $\mathbf{0 . 3}$ \\
D-49 & 347 & 1.3 & 0.0 & 2.0 & 1.2 & 0.9 & $\mathbf{5 . 4}$ \\
D-69 & 376 & 0.4 & 0.0 & 0.5 & 0.6 & 0.1 & $\mathbf{1 . 6}$ \\
D-99 & 397 & 1.7 & 0.0 & 2.3 & 0.2 & 0.3 & $\mathbf{4 . 5}$ \\
E-29 & 427 & 1.7 & 6.6 & 1.7 & 0.4 & 1.7 & $\mathbf{1 2 . 1}$ \\
E-51 & 449 & 2.8 & 0.3 & 1.9 & 0.0 & 0.1 & $\mathbf{5 . 1}$ \\
E-69 & 467 & 2.1 & 5.0 & 0.8 & 0.3 & 0.7 & $\mathbf{8 . 9}$ \\
E-99 & 497 & 2.8 & 0.6 & 2.7 & 0.0 & 0.8 & $\mathbf{6 . 9}$ \\
\hline
\end{tabular}

\section{Discussion}

Because of its greater sampling resolution and longer record, core 96-2 was used to define five ostracode zones (fig. 2). The zones are based solely on the stratigraphic structure of the various species. Because the fauna is largely endemic, the mechanisms responsible for the structure are poorly understood.

Zone $1(0$ to $87 \mathrm{~cm})$ is characterized by the highest total concentrations of ostracode valves in the core. Candona sp. 1, sp. 2, and sp. 3 are especially abundant. The unidentified species and Limnocythere sp. 1 are at their highest concentrations as well. Candona sp. 4 abundances decline upward through this interval.

Zone 2 ( 87 to $192 \mathrm{~cm}$ ) is defined by the near absence of Candona sp. 2 at 87 and $192 \mathrm{~cm}$. It also encompasses two discrete peaks of increased Candona sp. 2 concentrations at 110 and $155 \mathrm{~cm}$. Candona sp. 4 concentrations are near their maximum throughout this zone. Limnocythere sp. 1 becomes firmly established in this zone, and its concentrations increase upward through the zone. 

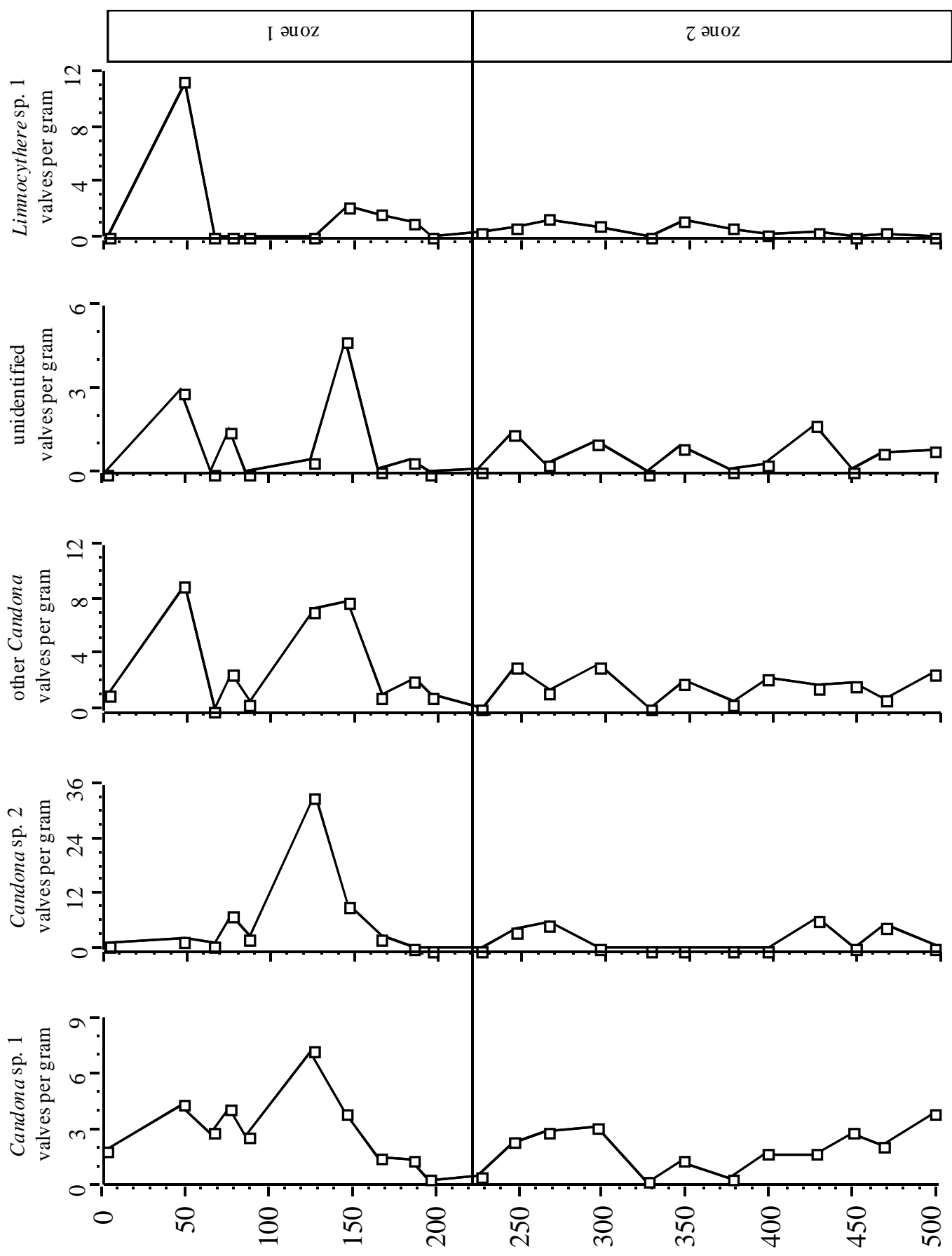

(யว) чमdәव

Figure 1. Abundances of common ostracode fauna (valves per gram, vpg) vs. depth for core 96-1. Faunal zones are shown on the right side of the diagram. 
Table 3. Ostracode concentrations in core 96-2.

Note: The core was cut into 5 sections, A through E. Sample numbers indicate the depth in each section, in centimeters, relative to the top of the core liner for that section. The Depth in the core is the depth relative to the uppermost sediments in section $A$.

\begin{tabular}{|c|c|c|c|c|c|c|c|c|c|c|c|c|}
\hline Sample No. & $\begin{array}{l}\text { Depth } \\
(\mathrm{cm})\end{array}$ & $\begin{array}{c}\text { Candona } \\
\text { sp. } 1 \\
\text { (valves/g) }\end{array}$ & $\begin{array}{c}\text { Candona } \\
\text { sp. } 2 \\
\text { (valves/g) }\end{array}$ & $\begin{array}{c}\text { Candona } \\
\text { sp. } 3 \\
\text { (valves/g) }\end{array}$ & $\begin{array}{c}\text { Candona } \\
\text { sp. } 4 \\
\text { (valves/g) }\end{array}$ & $\begin{array}{c}\text { Candona } \\
\text { sp. } 5 \\
\text { (valves/g) }\end{array}$ & $\begin{array}{c}\text { Candona } \\
\text { sp. } 6 \\
\text { (valves/g) }\end{array}$ & $\begin{array}{c}\text { Candona } \\
\text { sp. } 7 \\
\text { (valves/g) }\end{array}$ & $\begin{array}{c}\text { Limno. } \\
\text { sp. } 1 \\
\text { (valves/g) }\end{array}$ & $\begin{array}{c}\text { Limno. } \\
\text { sp. } 2 \\
\text { (valves/g) }\end{array}$ & $\begin{array}{l}\text { unidentified } \\
\text { species } \\
\text { (valves/g) }\end{array}$ & $\begin{array}{c}\text { TOTAL } \\
\text { (valves/g) }\end{array}$ \\
\hline A-11 & 1 & 7.3 & 60.0 & 3.6 & 0.9 & 1.8 & 0.9 & 0.0 & 0.9 & 0.0 & 0.0 & 83 \\
\hline A-16 & 6 & 1.0 & 13.0 & 1.0 & 0.0 & 0.0 & 0.0 & 0.0 & 0.0 & 0.0 & 0.0 & 15 \\
\hline A- 20 & 10 & 8.7 & 22.7 & 6.0 & 0.7 & 0.7 & 0.0 & 0.0 & 1.3 & 0.0 & 0.7 & 61 \\
\hline A-24 & 14 & 8.8 & 30.6 & 6.3 & 0.6 & 1.3 & 0.0 & 0.0 & 1.9 & 0.0 & 1.3 & 81 \\
\hline A- 28 & 18 & 11.2 & 40.6 & 1.2 & 1.2 & 1.8 & 0.0 & 0.0 & 1.8 & 0.0 & 0.0 & 98 \\
\hline A-32 & 22 & 20.0 & 126.3 & 1.3 & 0.0 & 1.3 & 2.5 & 1.3 & 1.3 & 0.0 & 0.0 & 123 \\
\hline A-36 & 26 & 14.1 & 50.6 & 6.8 & 0.0 & 1.8 & 0.0 & 0.6 & 2.1 & 0.0 & 3.2 & 269 \\
\hline A- -40 & 30 & 5.7 & 25.7 & 5.7 & 3.3 & 0.0 & 0.0 & 0.0 & 12.9 & 0.0 & 2.4 & 117 \\
\hline A- -44 & 34 & 7.3 & 50.9 & 7.3 & 0.0 & 0.0 & 0.0 & 0.0 & 0.0 & 0.0 & 0.0 & 72 \\
\hline A- -48 & 38 & 8.1 & 56.9 & 5.6 & 0.6 & 0.0 & 1.3 & 0.0 & 0.0 & 0.0 & 0.6 & 117 \\
\hline A-52 & 42 & 10.7 & 95.0 & 14.3 & 0.0 & 0.0 & 1.4 & 0.0 & 0.0 & 0.0 & 0.0 & 170 \\
\hline A-56 & 46 & 8.6 & 42.9 & 9.6 & 1.8 & 0.0 & 2.5 & 0.4 & 3.9 & 0.0 & 1.4 & 199 \\
\hline A- 60 & 50 & 5.8 & 45.8 & 5.8 & 0.0 & 0.0 & 1.7 & 0.0 & 0.0 & 0.0 & 0.8 & 72 \\
\hline A-64 & 54 & 8.6 & 69.5 & 8.6 & 1.9 & 0.0 & 2.4 & 0.0 & 2.4 & 0.0 & 1.0 & 198 \\
\hline A- 68 & 58 & 7.5 & 27.1 & 5.0 & 1.8 & 0.0 & 1.1 & 0.0 & 4.3 & 0.0 & 1.8 & 136 \\
\hline A-72 & 62 & 8.6 & 12.3 & 7.7 & 5.0 & 0.0 & 0.9 & 0.5 & 8.6 & 0.0 & 2.3 & 101 \\
\hline A-76 & 66 & 5.0 & 7.5 & 5.5 & 5.0 & 0.0 & 1.5 & 0.0 & 4.0 & 0.0 & 0.5 & 58 \\
\hline A- 80 & 70 & 2.0 & 10.0 & 4.5 & 3.5 & 0.0 & 1.5 & 0.0 & 1.5 & 0.0 & 1.0 & 48 \\
\hline A- 83 & 73 & 2.4 & 5.2 & 2.9 & 1.4 & 0.0 & 1.0 & 0.0 & 2.4 & 0.0 & 0.0 & 32 \\
\hline A-87 & 77 & 3.6 & 5.9 & 1.8 & 5.0 & 0.0 & 2.3 & 0.0 & 1.8 & 0.0 & 0.0 & 45 \\
\hline A-91 & 81 & 3.2 & 2.7 & 1.1 & 2.2 & 0.0 & 0.8 & 0.5 & 5.7 & 1.1 & 0.8 & 67 \\
\hline A-95 & 85 & 1.6 & 1.6 & 0.0 & 0.6 & 0.0 & 0.3 & 0.0 & 0.9 & 0.0 & 0.0 & 16 \\
\hline A-99 & 89 & 3.4 & 6.6 & 0.9 & 5.0 & 0.0 & 0.0 & 0.0 & 2.5 & 0.0 & 0.0 & 59 \\
\hline B-3 & 93 & 3.5 & 15.9 & 0.6 & 4.7 & 0.0 & 0.0 & 0.0 & 4.4 & 0.0 & 0.6 & 101 \\
\hline B-7 & 97 & 8.5 & 11.5 & 3.3 & 4.6 & 0.0 & 0.5 & 0.0 & 3.8 & 0.0 & 0.5 & 128 \\
\hline B-11 & 101 & 6.1 & 25.6 & 1.1 & 4.7 & 0.0 & 1.7 & 0.0 & 1.1 & 0.0 & 0.3 & 146 \\
\hline B-15 & 105 & 7.7 & 50.9 & 0.6 & 3.4 & 0.0 & 2.0 & 0.0 & 2.3 & 0.0 & 1.7 & 240 \\
\hline B-19 & 109 & 6.1 & 25.3 & 0.3 & 7.8 & 0.0 & 0.0 & 0.0 & 3.6 & 0.0 & 2.8 & 165 \\
\hline B-23 & 113 & 5.3 & 13.1 & 0.3 & 4.1 & 0.0 & 0.0 & 0.6 & 3.8 & 0.0 & 0.3 & 88 \\
\hline B-27 & 117 & 3.9 & 17.8 & 0.3 & 4.7 & 0.0 & 0.0 & 0.3 & 4.2 & 0.0 & 1.9 & 119 \\
\hline B-32 & 122 & 5.1 & 7.1 & 0.3 & 4.0 & 0.0 & 0.0 & 0.0 & 1.1 & 0.0 & 0.0 & 62 \\
\hline B-36 & 126 & 3.8 & 11.3 & 0.0 & 5.0 & 0.0 & 0.0 & 0.4 & 3.3 & 0.0 & 0.4 & 58 \\
\hline B-40 & 130 & 7.9 & 3.6 & 0.7 & 11.8 & 0.0 & 0.0 & 1.1 & 6.4 & 0.0 & 0.0 & 88 \\
\hline B-44 & 134 & 4.2 & 3.6 & 0.3 & 3.3 & 0.0 & 0.3 & 0.3 & 1.2 & 0.0 & 0.0 & 44 \\
\hline B- 48 & 138 & 3.0 & 5.7 & 0.9 & 6.5 & 0.0 & 0.0 & 0.0 & 0.4 & 0.0 & 0.4 & 39 \\
\hline B-52 & 142 & 3.2 & 13.2 & 5.3 & 3.7 & 0.5 & 0.5 & 0.0 & 2.1 & 0.0 & 0.0 & 54 \\
\hline B-56 & 146 & 3.3 & 13.3 & 1.8 & 2.4 & 0.6 & 0.0 & 0.0 & 0.6 & 0.0 & 0.0 & 73 \\
\hline B-60 & 150 & 6.5 & 51.0 & 7.5 & 2.0 & 0.0 & 0.0 & 0.0 & 0.0 & 0.0 & 1.0 & 136 \\
\hline B-64 & 154 & 10.7 & 54.4 & 3.0 & 3.0 & 0.0 & 0.0 & 0.0 & 1.1 & 0.0 & 0.4 & 196 \\
\hline B-68 & 158 & 5.7 & 23.2 & 1.1 & 0.7 & 0.0 & 0.0 & 0.4 & 0.0 & 0.0 & 0.0 & 87 \\
\hline B-72 & 162 & 4.2 & 28.2 & 2.4 & 3.3 & 1.5 & 0.3 & 0.0 & 0.0 & 0.0 & 0.6 & 134 \\
\hline B-76 & 166 & 11.1 & 18.6 & 2.1 & 7.9 & 0.0 & 1.4 & 0.0 & 1.1 & 0.0 & 0.0 & 118 \\
\hline B-80 & 170 & 10.0 & 10.0 & 2.9 & 5.7 & 0.0 & 0.0 & 0.0 & 0.0 & 0.0 & 0.0 & 80 \\
\hline
\end{tabular}


Table 3 (cont.). Ostracode concentrations in core 96-2. - Continued

\begin{tabular}{|c|c|c|c|c|c|c|c|c|c|c|c|c|}
\hline Sample No. & $\begin{array}{l}\text { depth } \\
(\mathrm{cm})\end{array}$ & $\begin{array}{c}\text { Candona } \\
\text { sp. } 1 \\
\text { (valves/g) }\end{array}$ & $\begin{array}{c}\text { Candona } \\
\text { sp. } 2 \\
\text { (valves/g) }\end{array}$ & $\begin{array}{c}\text { Candona } \\
\text { sp. } 3 \\
\text { (valves/g) }\end{array}$ & $\begin{array}{c}\text { Candona } \\
\text { sp. } 4 \\
\text { (valves/g) }\end{array}$ & $\begin{array}{c}\text { Candona } \\
\text { sp. } 5 \\
\text { (valves/g) }\end{array}$ & $\begin{array}{c}\text { Candona } \\
\text { sp. } 6 \\
\text { (valves/g) }\end{array}$ & $\begin{array}{c}\text { Candona } \\
\text { sp. } 7 \\
\text { (valves/g) }\end{array}$ & $\begin{array}{c}\text { Limno. } \\
\text { sp. } 1 \\
\text { (valves/g) }\end{array}$ & $\begin{array}{c}\text { Limno. } \\
\text { sp. } 2 \\
\text { (valves/g) }\end{array}$ & $\begin{array}{l}\text { unidentified } \\
\text { species } \\
\text { (valves/g) }\end{array}$ & $\begin{array}{c}\text { TOTAL } \\
\text { (valves/g) }\end{array}$ \\
\hline B-84 & 174 & 10.7 & 13.7 & 1.3 & 1.0 & 0.0 & 0.0 & 0.0 & 0.0 & 0.0 & 0.7 & 82 \\
\hline B- 88 & 178 & 8.4 & 4.8 & 0.0 & 2.9 & 0.0 & 0.0 & 0.3 & 0.0 & 0.0 & 0.0 & 51 \\
\hline B-92 & 182 & 10.0 & 20.4 & 1.8 & 1.1 & 0.4 & 2.1 & 0.4 & 0.0 & 0.0 & 0.4 & 102 \\
\hline B-96 & 186 & 3.3 & 14.6 & 0.0 & 0.0 & 0.0 & 0.0 & 0.4 & 0.0 & 0.0 & 0.0 & 44 \\
\hline B-100 & 190 & 3.8 & 1.5 & 0.0 & 0.3 & 0.0 & 0.0 & 1.5 & 0.3 & 0.0 & 0.3 & 30 \\
\hline $\mathrm{C}-3$ & 194 & 5.3 & 11.1 & 0.3 & 0.0 & 0.0 & 0.0 & 0.3 & 0.0 & 0.0 & 0.0 & 64 \\
\hline C-7 & 198 & 9.2 & 3.3 & 0.3 & 0.0 & 0.0 & 0.0 & 0.3 & 0.0 & 0.0 & 0.0 & 51 \\
\hline C-11 & 202 & 22.7 & 11.8 & 0.0 & 0.0 & 0.0 & 0.0 & 0.5 & 0.0 & 0.0 & 0.0 & 77 \\
\hline C-14 & 205 & 18.6 & 39.1 & 1.8 & 0.5 & 0.0 & 0.0 & 2.3 & 0.0 & 0.0 & 0.9 & 139 \\
\hline C-18 & 209 & 20.5 & 34.5 & 4.1 & 0.5 & 0.0 & 0.0 & 0.9 & 0.0 & 0.0 & 0.9 & 135 \\
\hline C- 22 & 213 & 20.8 & 53.3 & 2.1 & 0.0 & 0.0 & 0.0 & 0.8 & 0.0 & 0.0 & 1.7 & 189 \\
\hline C-26 & 217 & 4.3 & 11.4 & 1.0 & 0.5 & 0.0 & 0.0 & 0.5 & 1.0 & 0.0 & 0.0 & 39 \\
\hline C-30 & 221 & 10.0 & 16.5 & 0.0 & 0.0 & 0.5 & 0.0 & 0.5 & 1.5 & 0.0 & 0.0 & 58 \\
\hline C-34 & 225 & 5.9 & 5.5 & 0.0 & 1.8 & 0.0 & 0.0 & 0.0 & 0.0 & 0.0 & 0.0 & 29 \\
\hline C-38 & 229 & 9.1 & 5.9 & 0.0 & 0.9 & 0.0 & 0.0 & 0.0 & 0.5 & 0.0 & 0.0 & 36 \\
\hline C-41 & 232 & 12.7 & 4.5 & 0.0 & 0.9 & 0.0 & 0.0 & 0.0 & 1.4 & 0.0 & 0.0 & 43 \\
\hline C-45 & 236 & 15.7 & 0.9 & 0.4 & 1.3 & 0.0 & 0.0 & 0.0 & 1.3 & 0.0 & 0.0 & 45 \\
\hline C-50 & 241 & 3.0 & 0.4 & 0.0 & 0.0 & 0.4 & 0.0 & 0.0 & 0.0 & 0.0 & 0.4 & 10 \\
\hline C-54 & 245 & 4.3 & 4.8 & 0.0 & 0.0 & 0.0 & 0.0 & 0.0 & 0.0 & 0.0 & 0.0 & 21 \\
\hline C-58 & 249 & 5.7 & 3.9 & 0.0 & 0.0 & 0.0 & 1.3 & 0.0 & 0.0 & 0.0 & 0.0 & 25 \\
\hline C-62 & 253 & 5.7 & 6.1 & 0.0 & 0.0 & 0.0 & 1.7 & 0.0 & 0.0 & 0.0 & 0.0 & 31 \\
\hline C-66 & 257 & 1.2 & 4.8 & 0.0 & 0.0 & 0.0 & 0.0 & 0.0 & 0.0 & 0.0 & 0.0 & 15 \\
\hline C-70 & 261 & 1.3 & 1.3 & 0.0 & 0.0 & 0.0 & 0.0 & 0.0 & 0.0 & 0.0 & 0.0 & 6 \\
\hline C-74 & 265 & 0.9 & 0.9 & 0.0 & 0.0 & 0.0 & 0.0 & 0.0 & 0.0 & 0.0 & 0.0 & 4 \\
\hline C-78 & 269 & 0.8 & 0.4 & 0.0 & 0.0 & 0.0 & 0.0 & 0.0 & 0.0 & 0.0 & 0.0 & 3 \\
\hline C- 82 & 273 & 2.0 & 0.0 & 0.0 & 0.0 & 0.0 & 0.0 & 0.0 & 0.0 & 0.0 & 0.0 & 5 \\
\hline C-86 & 277 & 0.8 & 0.8 & 0.0 & 0.0 & 0.0 & 0.0 & 0.0 & 0.0 & 0.0 & 0.0 & 4 \\
\hline C-90 & 281 & 0.0 & 0.4 & 0.0 & 0.0 & 0.0 & 0.0 & 0.0 & 0.0 & 0.0 & 0.0 & 1 \\
\hline C-94 & 285 & 0.0 & 1.3 & 0.0 & 0.0 & 0.0 & 0.0 & 0.0 & 0.0 & 0.0 & 0.0 & 3 \\
\hline C-98 & 289 & 0.9 & 1.4 & 0.0 & 0.0 & 0.0 & 0.5 & 0.0 & 0.0 & 0.0 & 0.0 & 6 \\
\hline D-0 & 292 & 1.7 & 0.0 & 0.0 & 0.0 & 0.0 & 0.0 & 0.0 & 0.0 & 0.0 & 0.0 & 2 \\
\hline D-4 & 296 & 0.4 & 0.0 & 0.0 & 0.0 & 0.0 & 0.0 & 0.0 & 0.0 & 0.0 & 0.0 & 1 \\
\hline D-8 & 299 & 0.6 & 0.6 & 0.0 & 0.0 & 0.0 & 0.0 & 0.0 & 0.0 & 0.0 & 0.0 & 2 \\
\hline D-11 & 303 & 0.0 & 0.5 & 0.0 & 0.0 & 0.0 & 0.0 & 0.0 & 0.0 & 0.0 & 0.0 & 1 \\
\hline D-16 & 308 & 0.5 & 1.5 & 0.0 & 0.0 & 0.0 & 0.0 & 0.0 & 0.0 & 0.0 & 0.0 & 4 \\
\hline D-20 & 312 & 0.5 & 0.0 & 0.0 & 0.0 & 0.0 & 0.0 & 0.0 & 0.0 & 0.0 & 0.0 & 1 \\
\hline D-24 & 316 & 0.0 & 0.0 & 0.0 & 0.0 & 0.0 & 0.0 & 0.0 & 0.0 & 0.0 & 0.0 & 0 \\
\hline D-28 & 320 & 0.0 & 0.0 & 0.0 & 0.0 & 0.0 & 0.0 & 0.0 & 0.0 & 0.0 & 0.0 & 0 \\
\hline D-32 & 324 & 0.0 & 0.0 & 0.0 & 0.0 & 0.0 & 0.0 & 0.0 & 0.0 & 0.0 & 0.0 & 0 \\
\hline D-36 & 328 & 0.0 & 0.0 & 0.0 & 0.0 & 0.0 & 0.0 & 0.0 & 0.0 & 0.0 & 0.0 & 0 \\
\hline D-40 & 332 & 0.0 & 0.0 & 0.0 & 0.0 & 0.0 & 0.0 & 0.0 & 0.0 & 0.0 & 0.0 & 0 \\
\hline D-44 & 336 & 0.0 & 0.0 & 0.0 & 0.0 & 0.0 & 0.0 & 0.0 & 0.0 & 0.0 & 0.0 & 0 \\
\hline D-48 & 340 & 0.5 & 0.0 & 0.0 & 0.0 & 0.0 & 0.0 & 0.0 & 0.0 & 0.0 & 0.0 & 1 \\
\hline D-52 & 344 & 0.0 & 0.0 & 0.0 & 0.0 & 0.0 & 0.0 & 0.0 & 0.0 & 0.0 & 0.0 & 0 \\
\hline D-56 & 348 & 0.0 & 0.0 & 0.0 & 0.0 & 0.0 & 0.0 & 0.0 & 0.0 & 0.0 & 0.0 & 0 \\
\hline D-60 & 352 & 0.0 & 0.0 & 0.0 & 0.0 & 0.0 & 0.0 & 0.0 & 0.0 & 0.0 & 0.0 & 0 \\
\hline
\end{tabular}


Table 3 (cont.). Ostracode concentrations in core 96-2. - Continued

\begin{tabular}{|c|c|c|c|c|c|c|c|c|c|c|c|c|}
\hline Sample No. & $\begin{array}{l}\text { depth } \\
(\mathrm{cm})\end{array}$ & $\begin{array}{c}\text { Candona } \\
\text { sp. } 1 \\
\text { (valves/g) }\end{array}$ & $\begin{array}{c}\text { Candona } \\
\text { sp. } 2 \\
\text { (valves/g) }\end{array}$ & $\begin{array}{c}\text { Candona } \\
\text { sp. } 3 \\
\text { (valves/g) }\end{array}$ & $\begin{array}{c}\text { Candona } \\
\text { sp. } 4 \\
\text { (valves/g) }\end{array}$ & $\begin{array}{c}\text { Candona } \\
\text { sp. } 5 \\
\text { (valves/g) }\end{array}$ & $\begin{array}{c}\text { Candona } \\
\text { sp. } 6 \\
\text { (valves/g) }\end{array}$ & $\begin{array}{c}\text { Candona } \\
\text { sp. } 7 \\
\text { (valves/g) }\end{array}$ & $\begin{array}{c}\text { Limno. } \\
\text { sp. } 1 \\
\text { (valves/g) }\end{array}$ & $\begin{array}{c}\text { Limno. } \\
\text { sp. } 2 \\
\text { (valves/g) }\end{array}$ & $\begin{array}{l}\text { unidentified } \\
\text { species } \\
\text { (valves/g) }\end{array}$ & $\begin{array}{c}\text { TOTAL } \\
\text { (valves/g) }\end{array}$ \\
\hline D-64 & 356 & 0.0 & 0.0 & 0.0 & 0.0 & 0.0 & 0.0 & 0.0 & 0.0 & 0.0 & 0.0 & $\mathbf{0}$ \\
\hline D-68 & 360 & 0.0 & 0.0 & 0.0 & 0.0 & 0.0 & 0.0 & 0.0 & 0.0 & 0.0 & 0.0 & 0 \\
\hline D-72 & 364 & 0.0 & 0.0 & 0.0 & 0.0 & 0.0 & 0.0 & 0.0 & 0.0 & 0.0 & 0.0 & $\mathbf{0}$ \\
\hline D-76 & 368 & 0.0 & 0.0 & 0.0 & 0.0 & 0.0 & 0.0 & 0.0 & 0.0 & 0.0 & 0.0 & $\mathbf{0}$ \\
\hline D-80 & 372 & 0.0 & 0.0 & 0.0 & 0.0 & 0.0 & 0.0 & 0.0 & 0.0 & 0.0 & 0.0 & $\mathbf{0}$ \\
\hline D-84 & 376 & 0.0 & 0.0 & 0.0 & 0.0 & 0.0 & 0.0 & 0.0 & 0.0 & 0.0 & 0.0 & $\mathbf{0}$ \\
\hline D-88 & 380 & 0.0 & 0.0 & 0.0 & 0.0 & 0.0 & 0.0 & 0.0 & 0.0 & 0.0 & 0.0 & $\mathbf{0}$ \\
\hline D-92 & 384 & 0.0 & 0.0 & 0.0 & 0.0 & 0.0 & 0.0 & 0.0 & 0.0 & 0.0 & 0.0 & $\mathbf{0}$ \\
\hline D-96 & 388 & 0.0 & 0.0 & 0.0 & 0.0 & 0.0 & 0.0 & 0.0 & 0.0 & 0.0 & 0.0 & 0 \\
\hline D-100 & 392 & 0.0 & 0.0 & 0.0 & 0.0 & 0.0 & 0.0 & 0.0 & 0.0 & 0.0 & 0.0 & $\mathbf{0}$ \\
\hline
\end{tabular}

Zone $3(192$ to $255 \mathrm{~cm})$ is characterized by a large increase in the concentration of Candona sp. 1 and contains peaks in the concentrations of Candona sp. 2, Candona sp. 3, Candona sp. 7, and the unidentified species make their first appearances in the core and exhibit minor peaks in their concentrations in this zone. Candona sp. 4 occurs in low concentrations. Limnocythere sp. 1 has two small, isolated occurrences in this zone.

Zone 4 (255 to $308 \mathrm{~cm}$ ) contains low concentrations of Candona sp. 1 and Candona sp. 2.

Zone 5 (308 to $392 \mathrm{~cm}$ ) contains two isolated occurrences of Candona sp. 1; otherwise, it is barren.

Core 96-1 contains only two zones, which are equivalent to Zone 1 and the upper part of Zone 2 defined in core 96-2.

Zone $1(0$ to $221 \mathrm{~cm})$ is characterized by the highest ostracode concentrations in the core.

Zone $2(221$ to $500 \mathrm{~cm})$ is defined by overall low ostracode concentrations.

Correlating the minor fluctuations in ostracode assemblages in Zone 1 between the two cores is hampered by the low sample resolution in core 96-1. However, the overall ostracode concentration structure from core 96-1 seems to match well with the concentration structure in the upper $\sim 200 \mathrm{~cm}$ of core $96-2$ (fig. 3). Plotting the total ostracode concentrations by age (based on the age equations of Colman and others, 2005) shows that the overall rise and fall of ostracode concentrations are similar in the two cores (fig. 4). Both cores show a marked increase in ostracode concentrations at $\sim 3300$ cal yr BP. 

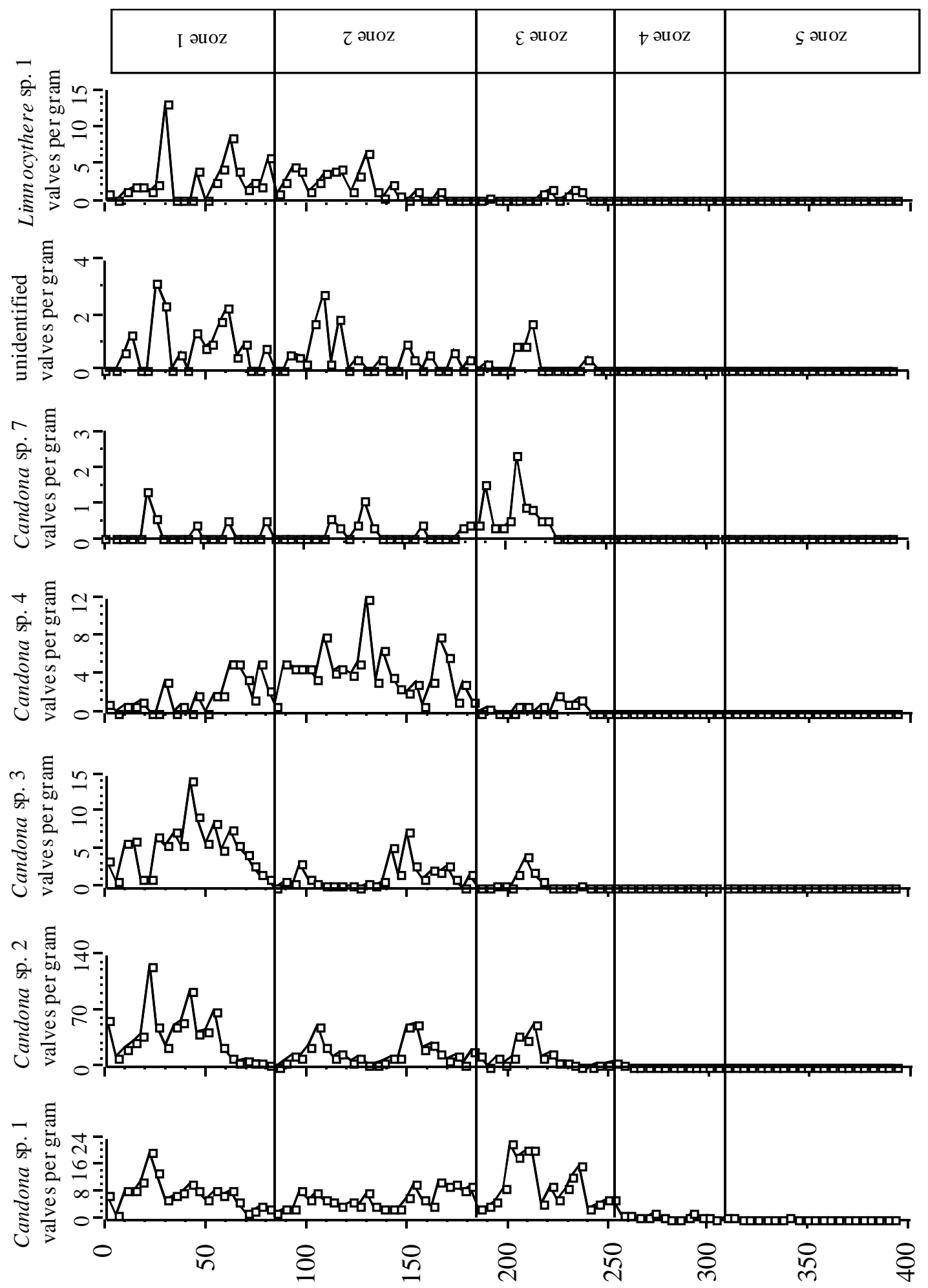

(யว) чрдә

Figure 2. Abundances of common ostracode fauna (valves per gram, vpg) vs. depth for core 96-2. Faunal zones are shown on the right side of the diagram. 


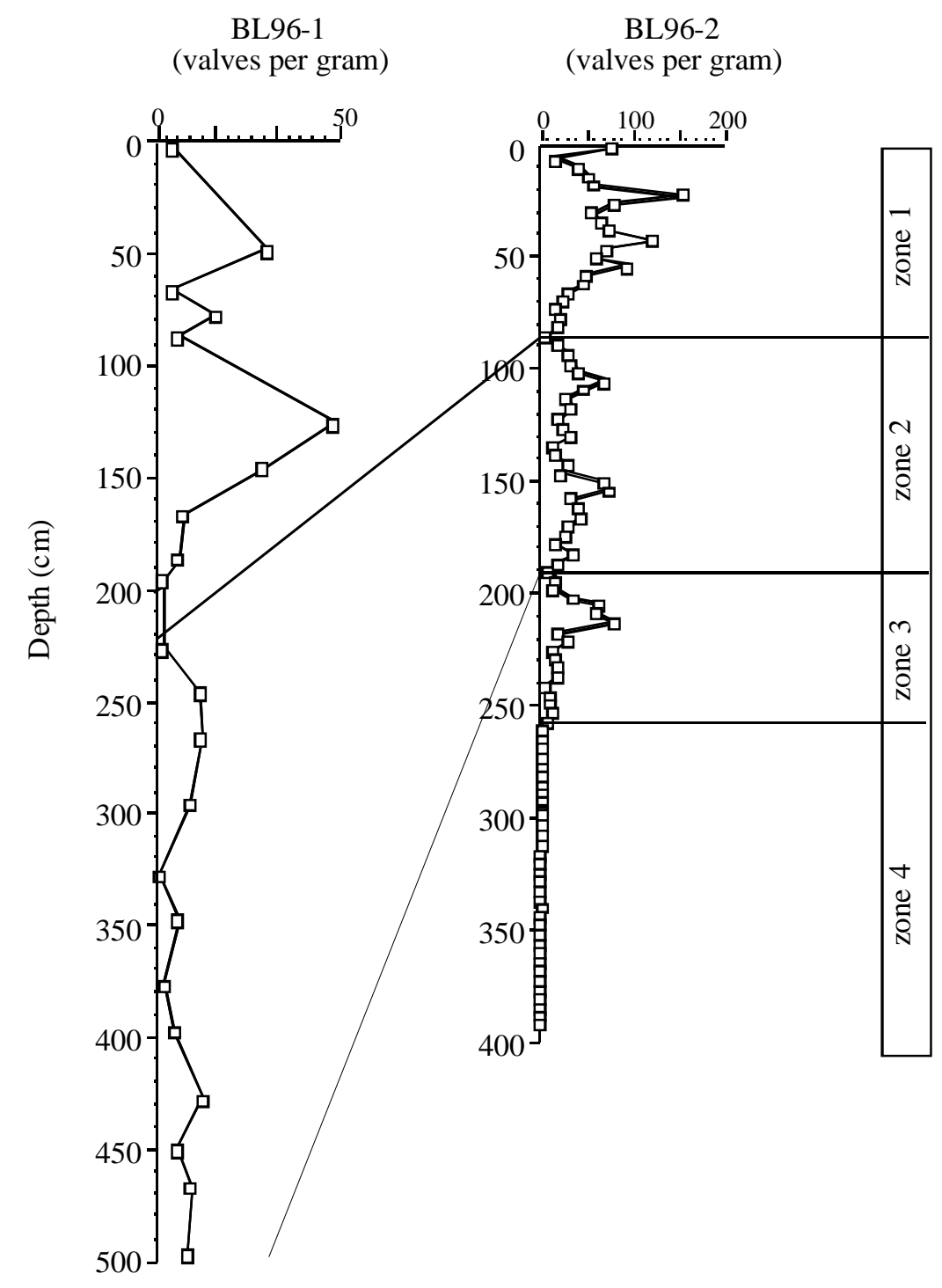

Figure 3. Correlation between ostracode zones vs. depth in cores 96-1 and 96-2, based on total valve concentrations (valves per gram, vpg). Faunal zones are shown on right side of diagram. 
BL96-2

(valves per gram)

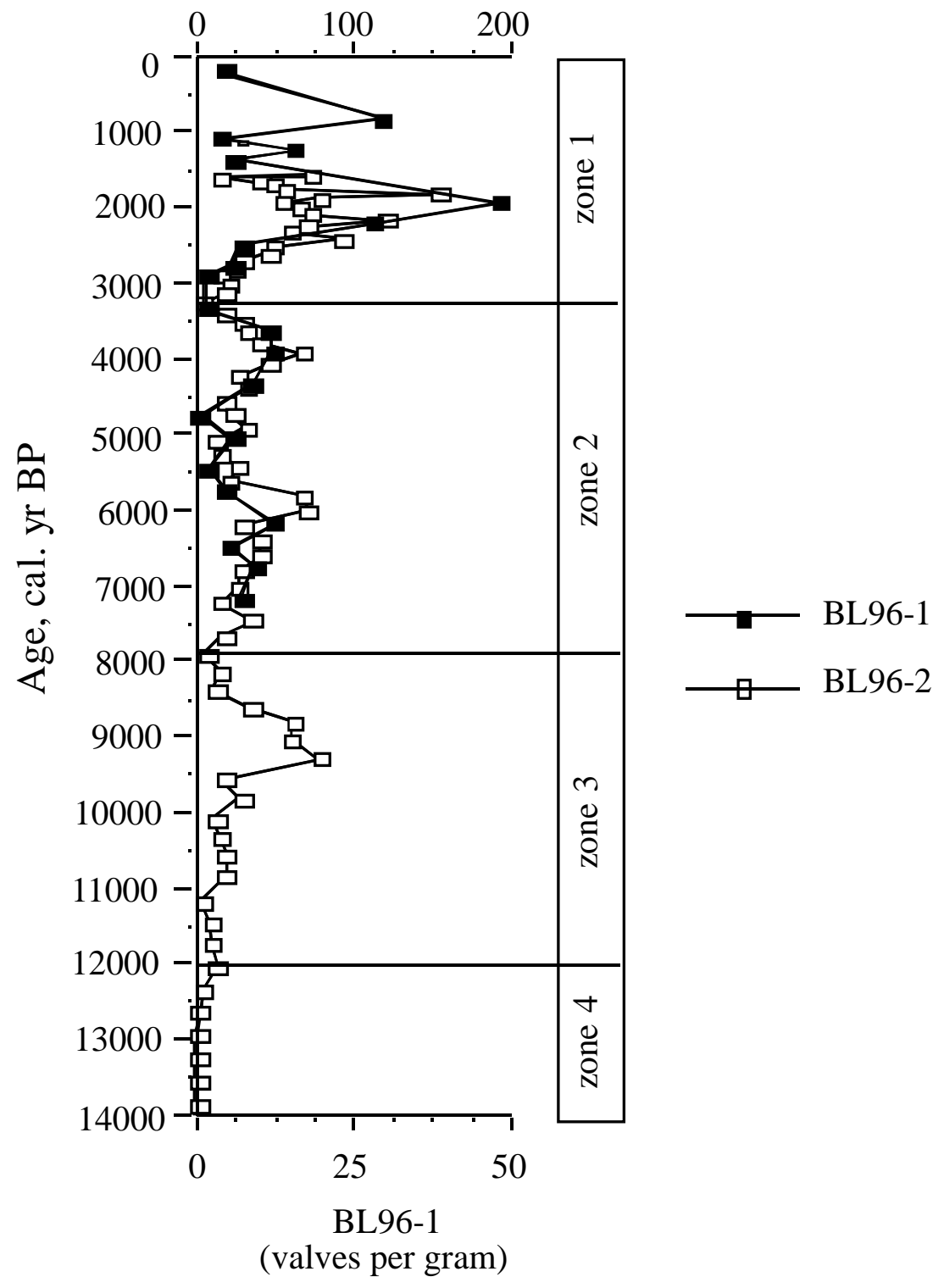

Figure 4. Total concentration of ostracodes (valves per gram, vpg) and faunal zones vs. age. Chronology from Colman and others (2005). Faunal zones are shown on right side of diagram. 


\section{References cited}

Colman, S., Kaufman, D., Rosenbaum, J.G., and McGeehin, 2005, Radiocarbon dating of cores collected from Bear Lake, Utah and Idaho: U.S. Geological Survey Open-File Report 2005-XXXX, Xp.

Dean W., Forester, R., Colman, S., Liu, A., Skipp, G., Simmons, K. Swarzenski, P., Anderson, R., 2005, Modern and glacial - Holocene carbonate sedimentation in Bear Lake, Utah and Idaho: U. S. Geological Survey Open File Report 2005-1124.

Denny, J.F., Colman, S.M., 2003, Geophysical surveys of Bear Lake, Utah-Idaho, September 2002: U. S. Geological Survey Open File Report 03-150.

Dettman, D.L, Smith, A.J., Rea, D.K., Moore, T.C. Jr., and Lohmann, K.C., 1995, Glacial meltwater in Lake Huron during early postglacial time as inferred from single-valve analysis of oxygen isotopes in ostracodes: Quaternary Research, v. 43, p. 297-310.

Forester, R.M., Colman, S.M., Reynolds, R.L., and Kegwin, L.D., 1994, Lake Michigan's late Quaternary limnological and climate history from ostracode, oxygen isotope, and magnetic susceptibility: Journal of Great Lakes Research, v. 20(1), p. 93107.

Forester, R.M., 1987, Late Quaternary paleoclimate records from lacustrine ostracodes, In "North America and Adjacent Oceans During the Last Deglaciation" (W.F. Ruddiman and H.E. Wright, eds.), p. 261-276, The Geology of North America, v. K-3, The Geological Society of America, Boulder, Colorado.

Kaufman, D., 2003,. Dating deep-lake sediments by using amino acid racemization in fossil ostracodes: Geology, v. 31, p. 10491052.

Schwalb, A., Locke, S.M., and Dean, W.E., 1995, Ostracode $\delta^{18} 0$ and $\delta^{13} \mathrm{C}$ evidence of Holocene environmental changes in the sediments of two Minnesota lakes: Journal of Paleolimnology, v. 14, p. 281-296.

Smith, A.J., 1993, Lacustrine ostracodes as hydrochemical indicators in lakes of the north-central United States: Journal of Paleolimnology, v. 8, p. 121-134.

Wells, T.M., Cohen, A.S., Park, L.E., Dettman, D.L., and McKee, B.A., 1999, Ostracode stratigraphy and paleoecology from surficial sediments of Lake Tanganyika, Africa: Journal of Paleolimnology, v. 22, p. 259-276. 